\title{
Stimulation of tolerogenic dendritic cells using dexamethasone and 1,25-dihydroxyvitamin D3 represses autologous $T$ cell activation and chondrocyte inflammation
}

\author{
GAOYUAN WANG ${ }^{1}$, JUNQIANG ZHANG ${ }^{2-4}$, YUAN FANG $^{5}$, WEI CAO ${ }^{6}$, BIN XU $^{1}$ and XIAOYU CHEN ${ }^{6}$ \\ ${ }^{1}$ Department of Orthopaedics, The First Affiliated Hospital of Anhui Medical University, Hefei, Anhui 230031; \\ ${ }^{2}$ Anhui Province Key Laboratory of Reproductive Health and Genetics, Anhui Medical University, Hefei, \\ Anhui 230032; ${ }^{3}$ Reproductive Medicine Center, Department of Obstetrics and Gynecology, The First Affiliated Hospital of \\ Anhui Medical University, Hefei, Anhui 230031; ${ }^{4}$ Anhui Provincial Engineering Technology Research Center for \\ Biopreservation and Artificial Organs, Anhui Medical University, Hefei, Anhui 230032; ${ }^{5}$ Department of \\ Blood Transfusion, Anhui No. 2 Provincial People's Hospital, Hefei, Anhui 230041; ${ }^{6}$ Department of \\ Histology and Embryology, Anhui Medical University, Hefei, Anhui 230032, P.R. China
}

Received February 2, 2018; Accepted November 1, 2018

DOI: $10.3892 /$ etm.2018.7036

\begin{abstract}
Human osteoarthritis (OA) has been reclassified as a systemic musculoskeletal disorder involving activation of the innate and adaptive immune systems. Elevated pro-inflammatory cytokines may serve a key function in the development of the disease. 1,25-Dihydroxyvitamin D3 and dexamethasone (vitD3/Dex) may inhibit inflammation by acting on tolerogenic dendritic cells (tolDCs) in chronic inflammatory conditions. In the present study, DCs were isolated from peripheral blood mononuclear cells of patients with OA. DCs expressing high levels of co-stimulatory molecules maintain a tolerogenic phenotype under stimulation with LPS, which promotes DC maturation to generate tolDCs. When vitD3/Dex were added in the current study, the tolDCs produced pro-inflammatory cytokines, including low levels of tumor necrosis factor- $\alpha$, interleukin (IL)-1 $\beta$, IL-6 and IL-10, and high levels of transforming grow th factor- $\beta$. However, when vitD3/Dex were added to DCs without LPS stimulation, the levels of IL-10 were high. DCs with LPS stimulation increased the percentage of T-cells that produced IFN- $\gamma$ and IL-17 and DCs with vitD3/Dex treatment alone increased the percentage of T-cells that produced IL-10 and FoxP3. However, those cytokines decrease in DCs co-processed with LPS and vitD3/Dex. The IL-10 release by the stimulated $\mathrm{T}$ cells was indicated to repress autologous $\mathrm{T}$ cell proliferation via soluble IL-10 and cell-cell contact. Furthermore, tolDCs and regulatory T cells suppressed matrix
\end{abstract}

Correspondence to: Professor Xiaoyu Chen, Department of Histology and Embryology, Anhui Medical University, 81 Meishan Road, Hefei, Anhui 230032, P.R. China

E-mail: cxyayd@163.com

Key words: dendritic cell, regulatory T cell, osteoarthritis, vitamin D3, chondrocyte metalloproteinase (MMP)-1 and MMP-13 secretion by chondrocytes. Additionally, Akt and p38 mitogen-activated protein kinase signaling were demonstrated to be involved in the regulatory effects of Dec and vitD3 in DCs. The present findings suggest a novel mechanism underlying the beneficial effects of tolDCs, particularly in association with the pathogenesis of OA.

\section{Introduction}

Osteoarthritis (OA) is characterized by excessive production of cytokines and metalloproteinases, resulting in the degeneration of articular cartilage tissue (1). During the last century, it was believed that human osteoarthritis was a 'wear-and-tear' mechanically-driven focal musculoskeletal disorder associated with aging, and no medical treatment provided a 'cure', aside from arthroplasty (2). However, over 10 years ago, when Pelletier et al (3) re-conceptualized OA as an arthritis joint disease, its inflammation was deemed 'non-classic'. OA can occur in any joint, but predominantly occurs in the knees, hips, hands and spine (4). The main features of OA are joint cavity stenosis, subchondral bone remodeling, synovitis and cartilage degeneration (5). OA is the most common type of arthritis, and its incidence is associated with age, sex, obesity and joint injury (6). The incidence of OA is increasing (7). Therefore the demand for diagnosis and treatment of the disease is also increasing.

Matrix metalloproteinase (MMP)-1 and MMP-13, and A disintegrin and metalloproteinase with thrombospondin motifs (ADAMTS)-4 and ADAMTS-5 can degrade the extracellular cartilage matrix (8). During joint development in adults, chondrocytes promote the mineralization of cartilage through a final differentiation step, similar to the process of bone $f$ formation (9). Pro-inflammatory cytokines are vital mediators that lead to metabolic disorder and increased catabolism of joint tissue associated with OA (10). Interleukin (IL)-1 $\beta$, tumor necrosis factor- $\alpha(\mathrm{TNF}-\alpha)$ and IL6 are considered to be the 
major pro-inflammatory cytokines involved in the pathophysiology of OA (11).

Vitamin D has been well researched for its effects on calcium metabolism, and has also been reported to have a significant immunomodulatory effect. For instance, treatment of dendritic cells (DCs) with 1,25-dihydroxyvitamin D3 [1,25(OH) 2 D3] (vitD3) inhibited lipopolysaccharide (LPS)-induced inflammation (12). LPS has been demonstrated to promote DC maturation, which generates tolerogenic DCs (tolDCs), a maturation-resistant form of the cells with tolerogenic function $(11,13)$. Characteristics of tolDCs include high expression of co-stimulatory molecules and major histocompatibility complex (MHC) class II, and low production of pro-inflammatory cytokines, such as IL-12, IL-6 and TNF- $\alpha$ (14). tolDCs have been increasingly studied as a cell-based treatment and have produced promising results in mouse models of autoimmune diseases, including diabetes and inflammatory arthritis (15). They can induce and maintain peripheral $\mathrm{T}$ cell tolerance through multiple mechanisms, including induction of $\mathrm{T}$ cell deletion, anergy, cytokine deviation and induction of regulatory $\mathrm{T}$ cells (Tregs) (16). In the current study, DCs from patients with OA were treated with dexamethasone (Dex)/vitD3 and their phenotype and function as tolDCs was assessed to determine whether the protein kinase B (Akt) and p38 mitogen-activated protein kinase (MAPK) signaling pathways were involved in the induction of tolDCs when stimulated with Dex and vitD3.

\section{Materials and methods}

Patients. A total of 30 patients with OA (57-75 years old) were enrolled in the study, of which 17 were female and 13 male. The OA subjects were diagnosed according to the Western Ontario McMaster University Osteoarthritis Index (17), and the study was conducted by the First Affiliated Hospital of Anhui Medical University, Hefei, China. Clinical and laboratory examinations were performed after obtaining informed written consent from the OA patients from January 2017 to January 2018. The inclusion criteria for the diagnosis of OA were as follows: i) $\sim 1$ month of repeated joint pain with $>15$ incidents of knee pain; ii) having bone fricative; iii) morning stiffness lasting <30 min; iv) age at diagnosis >38 years; v) presentation of bony enlargement(s). Subjects exhibited some associated complications, including joint pain, tenderness, stiffness, joint effusion, limited mobility, joint deformities and local inflammation of varying degrees; this was in accord with the general characteristics of OA (17). Excluded patients were those with rheumatoid arthritis or gout-induced arthritis. The patients were not receiving any treatments prior to diagnosis. The study was approved by the Ethics Committee of Anhui Medical University.

Generation of Dex/vitD3-treated DCs. Peripheral blood mononuclear cells (PBMCs) and cluster of differentiation CD14 ${ }^{+}$ monocytes were separated from $5 \mathrm{ml}$ fresh venous blood by density centrifugation using Ficoll-Paque (GE Healthcare Life Sciences, Shanghai, China) and magnetic microbeads (Miltenyi Biotec GmbH, Bergisch Gladbach, Germany), respectively. In the presence of $50 \mathrm{ng} / \mathrm{ml}$ granulocyte-macrophage colony-stimulating factor and $25 \mathrm{ng} / \mathrm{ml} \mathrm{IL-4} \mathrm{(PeproTech,} \mathrm{Inc.,}$
Rocky Hill, NJ, USA), monocyte-derived immature DCs were produced by culture of monocytes at $1 \times 10^{6}$ cells $/ \mathrm{ml}$ for 7 days, and the medium was refreshed or pretreatment performed on day 4. Mature DCs were generated by addition of $100 \mathrm{ng} / \mathrm{ml}$ LPS (Sigma-Aldrich; Merck KGaA, Darmstadt, Germany) on day 6 for $24 \mathrm{~h}(\mathrm{DC}+\mathrm{LPS})$. Dex/vitD3-DCs were generated by adding $1 \mu \mathrm{M}$ Dex to DCs on day 4 , and $1 \mu \mathrm{M}$ Dex plus $0.1 \mathrm{nM}$ vitD3 on day 5, followed by addition of $100 \mathrm{ng} / \mathrm{ml} \mathrm{LPS}$ on day 6 for 24 h. Cells were cultured in RPMI-1640 medium (Hyclone; GE Healthcare Life Sciences, Logan, UT, USA) containing $10 \%$ fetal bovine serum (FBS, Gibco; Thermo Fisher Scientific, Inc., Waltham, MA, USA), 2 mM glutamine, and $1 \%$ penicillin and streptomycin at $37^{\circ} \mathrm{C}$ with $5 \% \mathrm{CO}_{2}$.

$D C-T$ cell co-culture experiments. $\mathrm{CD}^{+}$naive $\mathrm{T}$ cells $\left(\mathrm{CD}^{+} \mathrm{CD}^{4} 5 \mathrm{RA}^{+} \mathrm{CD} 45 \mathrm{RO}^{-}\right)$were separated using an Untouched $\mathrm{CD}^{+} \mathrm{T}$ Cell kit (Miltenyi Biotec $\mathrm{GmbH}$ ). A total of $1 \times 10^{5}$ autologous DCs were co-cultured with $1 \times 10^{6}$ autologous naive T cells (1:10) in six well plates from the same individual. Cells were cultured in RPMI-1640 containing 10\% FBS, 2 mM glutamine, and $1 \%$ penicillin and streptomycin at $37^{\circ} \mathrm{C}$ with $5 \% \mathrm{CO}_{2}$ for 5 days. Assessment of $\mathrm{T}$ cell proliferation was performed with $1 \mu \mathrm{Ci} /$ well $\left[{ }^{3} \mathrm{H}\right]$-thymidine in the last $16 \mathrm{~h}$ of culture using scintigraphy (PerkinElmer, Inc., Waltham, MA, USA).

$T$ cell differentiation. $\mathrm{CD}^{+}$naive $\mathrm{T}$ cells $\left(1 \times 10^{6}\right)$ were co-cultured with $1 \times 10^{5}$ autologous DCs in $1 \mathrm{ml}$ of X-vivo 15 medium (Lonza Group, Ltd., Basel, Switzerland) for 6 days at $37^{\circ} \mathrm{C}$ with $5 \% \mathrm{CO}_{2}$. Recombinant human IL-2 (rhIL-2; $20 \mathrm{U} / \mathrm{ml}$; cat. no. CTP0021; Thermo Fisher Scientific, Inc.) was added on day 6 , and cells were cultured for a further 6 days. $\mathrm{T}$ cells were collected after 12 days, then washed, and their cellular functions were analyzed. T cells co-cultured with immature DCs were termed T [DC], co-cultured with mature DCs as T [DC + LPS], co-cultured with Dex/vitD3-DCs as $\mathrm{T}[\mathrm{DC}+\mathrm{Dex} / \mathrm{vitD} 3]$ and co-cultured with LPS-induced Dex/vitD3-DCs as T [DC + Dex/vitD3 + LPS].

T cell proliferation and suppression. To assess the ability of these $\mathrm{T}$ cells to inhibit proliferation and/or cytokine generation, allogeneic mature DCs $\left(10: 1 ; 1 \times 10^{4} \mathrm{~T}\right.$ cells: $\left.1 \times 10^{3} \mathrm{DCs}\right)$ were used to stimulate $\mathrm{CD}^{+}{ }^{+}$naive $\mathrm{T}$ cells with or without autologous T [DC], T [DC + LPS $], T$ [DC + Dex/vitD3)] or $\mathrm{T}[\mathrm{DC}+\mathrm{Dex} / \mathrm{vitD} 3+\mathrm{LPS})]$ cells (1:1 ratio) in a final volume of $200 \mu \mathrm{l}$ RPMI-1640 containing $10 \%$ FBS in 96-well plates. Neutralizing anti-IL-10 receptor (IL-10R; 1:200 dilution; cat. no. 556012; BD Biosciences, San Jose, CA, USA), anti-IL-10 (1:200 dilution; cat. no. 554703; BD Pharmingen; BD Biosciences) or anti-transforming growth factor- $\beta$ (TGF- $\beta$; 1:300 dilution; cat. no. MAB1835-100; R\&D Systems, Inc., Minneapolis, MN, USA) monoclonal antibodies were added into cultures. Cells were cultured at $37^{\circ} \mathrm{C}$ with $5 \% \mathrm{CO}_{2}$ for 5 days with $1 \mu \mathrm{Ci} /$ well $\left[{ }^{3} \mathrm{H}\right]$-thymidine was added to the cultures in the last $16 \mathrm{~h}$ for measurement of proliferation by scintigraphy.

Chondrocyte isolation and identification. Cartilage specimens were acquired from the femoral condyles of the aformentioned patients with knee OA that had undergone total knee arthroplasty between January 2017 and 2018. The fragments were 
washed thoroughly in Dulbecco's modified Eagle's medium (DMEM; Thermo Fisher Scientific, Inc.) containing 1\% penicillin/streptomycin solution (Sigma-Aldrich; Merck KGaA), then samples were cut into small pieces and digested with various enzymes. The tissue pieces were treated with $0.1 \%$ hyaluronidase for $20 \mathrm{~min}, 0.5 \%$ pronase for $1 \mathrm{~h}$ and $0.2 \%$ collagenase for $1 \mathrm{~h}$ (Gibco; Thermo Fisher Scientific, Inc.) at $37^{\circ} \mathrm{C}$. Subsequently, the cell suspension was filtered through a 100- $\mu \mathrm{m}$ Celltrics filter (EMD Millipore, Billerica, MA, USA), washed and then centrifuged at $200 \mathrm{x} \mathrm{g}$ for $10 \mathrm{~min}$ at $4^{\circ} \mathrm{C}$. Human primary chondrocytes were cultured with DMEM containing $10 \% \mathrm{FBS}$ at $37^{\circ} \mathrm{C}$ in a $5 \% \mathrm{CO}_{2}$ incubator for 2 weeks. The culture medium was replenished every 5 days. Chondrocytes were seeded on glass coverslips in 6-well plates at $1 \times 10^{5}$ cells/well and then harvested. The sections were washed 2-3 times, and then were stained with $1 \%$ toluidine blue for $10 \mathrm{~min}$ at room temperature, and then observed under an optical microscope. The coverslips with the chondrocytes were fixed with $4 \%(\mathrm{v} / \mathrm{v})$ paraformaldehyde for $30 \mathrm{~min}$ at $37^{\circ} \mathrm{C}$, then permeablized with $0.1 \%$ Triton X-100 for $20 \mathrm{~min}$ at room temperature, washed three times and incubated with anti-collagen II antibody (1:300 dilution; cat. no. Ab34712; Abcam, Cambridge, MA, USA) overnight at $4^{\circ} \mathrm{C}$. The subsequent day, horseradish peroxidase-labeled secondary antibodies (1:100; cat. no. A0208; Beyotime Institute of Biotechnology, Haimen, China) was added for $1 \mathrm{~h}$ at $37^{\circ} \mathrm{C}$. This was followed by immunostaining with 3,3-diaminobenzidine tetrahydrochloride for 1-3 $\mathrm{min}$ at room temperature. The coverslips were counterstained with hematoxylin for 30-60 sec at room temperature, and then rinsed under light running water. Images of these cells were captured at x200 magnification in light microscope (Olympus IX73; Olympus Corporation, Tokyo, Japan).

DC-chondrocyte or T cell-chondrocyte co-culture experiments. An aliquot of first generation chondrocytes was seeded in 24 -well plates at $5 \times 10^{4}$ cells per well. Cells cultured with $20 \mathrm{ng} / \mathrm{ml} \mathrm{TNF}-\alpha$ for $12 \mathrm{~h}$ were rinsed, and co-cultured with the autologous DC or Tregs at a ratio of 10:1 (chondrocytes: DC or $\mathrm{T}$ cells) in DMEM containing $10 \% \mathrm{FBS}$ at $37^{\circ} \mathrm{C}$ in a $5 \% \mathrm{CO}_{2}$ incubator for $24 \mathrm{~h}$.

Flow cytometry. DCs or T cells were incubated with Human TruStain FcX ${ }^{\mathrm{TM}}$ (Fc Receptor Blocking Solution; Miltenyi Biotec $\mathrm{GmbH}$ ) for $10 \mathrm{~min}$, and then with primary antibodies on ice for $30 \mathrm{~min}$. Human leukocyte antigen-antigen D related-phycoerythrin (HLA-DR-PE; cat. no. 560943), CD86-fluorescein isothiocyanate (FITC; cat. no. 560958), CD40-peridinin chlorophyll protein complex-cyanin 5 (Pe-Cv5; cat.no.560963), CD83-allophycocyanin and isotypic controls (APC; cat. no. 561960; all BD Biosciences) were used for flow cytometry. The intracellular cytokine profile was assessed using the monoclonal antibodies interferon- $\gamma$ (IFN- $\gamma$ )-FITC (cat. no. 552882), FoxP3-Alexa-647 (cat. no. 561184), IL-10-PE (cat. no. 559330) and IL-17-PE (cat. no. 560486; all BD Biosciences). A total of $1 \times 10^{6}$ cells were permeabilized and fixed using $30 \mu \mathrm{l}$ cytofix/cytoperm buffers (eBioscience; Thermo Fisher Scientific, Inc.) for $30 \mathrm{~min}$ at $4^{\circ} \mathrm{C}$. Then the cells were resuspended in $200 \mu \mathrm{l}$ antibody solution (1:200) and $30 \mu 1 \mathrm{lX}$ Perm/Wash buffer (cat. no. 554723; BD Biosciences). The cells were incubated with the antibodies for $30 \mathrm{~min}$ at $4^{\circ} \mathrm{C}$ and wash twice in $200 \mu 1$ 1X Perm/Wash Buffer. Golgistop (1:1,500 dilution; cat. no. 554724; BD Biosciences) was added at $37^{\circ} \mathrm{C}$ in a $5 \%$ $\mathrm{CO}_{2}$ incubator for 4-6 h. Samples were analyzed by flow cytometry (BD FACSCalibur; BD Biosciences) and results were analyzed using FlowJo software 7.6.2 (FlowJo LLC, Ashland, OR, USA).

Phospho flow cytometry. Cells $\left(1 \times 10^{6}\right)$ were fixed with $4 \%$ paraformaldehyde for $15 \mathrm{~min}$ at $37^{\circ} \mathrm{C}$, then washed three times, permeabilized with cytofix/cytoperm buffers for $30 \mathrm{~min}$ on ice and stained with either anti-Akt (pS473)-Alexa Fluor ${ }^{\circledR} 488$ (cat. no. 560404) or with anti-p38 MAPK (pT180/pY182)-Alexa Fluor 647 (cat. no. 612595; both 1:200; BD Biosciences) for $30 \mathrm{~min}$ at $4^{\circ} \mathrm{C}$. Flow cytomery analysis was performed as previously described.

ELISA. Supernatants were harvested from DC cultures, DC or $\mathrm{T}$ cell-chondrocyte co-cultures and stored at $-80^{\circ} \mathrm{C}$ for subsequent cytokine measurements. Cytokines concentations were measured by ELISA using the following kits: human TNF- $\alpha$ (cat. no. DTA00C), human TGF- $\beta$ (cat. no. DB100B), human IL1 $\beta$ (cat. no. DLB50), human IL-6 (cat. no. D6050), human IL-10 kit (cat. no. D1000B), MMP-1 (cat. no. DMP100) and MMP-13 (cat. no. DM1300; all R\&D Systems, Inc.) according to the manufacturer's protocol. Absorbance was determined using a Thermo Scientific Microplate Reader (Thermo Fisher Scientific, Inc.).

Reverse transcription-quantitative polymerase chain reaction $(R T-q P C R)$. Total RNAs were extracted from DC-primed naive $\mathrm{T}$ cells using TRIzol ${ }^{\circledR}$ Reagent (Sigma-Aldrich; Merck KGaA). First-strand cDNAs were then synthesized using a SuperScript ${ }^{\circledR}$ III Reverse Transcriptase kit (Invitrogen; Thermo Fisher Scientific, Inc.) and then converted into cDNA on a Mastercycler ${ }^{\circledR}$ nexus (Eppendorf, Hamburg, Germany) using a PrimeScript ${ }^{\circledR}$ RT reagent kit (Takara Bio, Inc., Otsu, Japan), according to the manufacturer's protocol. qPCR was conducted using SYBR Green PCR Master mix (with Rox; Invitrogen; Thermo Fisher Scientific, Inc.) on LightCycler ${ }^{\circledR}$ 480 (Roche Diagnostics, Basel, Switzerland). $\beta$-actin was used as an internal reference for mRNA expression. Primer sequences were as follows: T-box 21 (T-bet), forward, 5'-GGT AACATGCCAGGGAACAGGA-3' and reverse, 5'-TGG TCTATTTTTAGCTGGGTGATGTCTG-3'; GATA binding protein 3 (Gata-3), forward, 5'-CCAAAAACAAGGTCATGT TCAGAAGG-3' and reverse, 5'-TGGTGAGAGGTCGGTTGA TATTGTG-3'; forkhead box P3 (Foxp3), forward, 5'-GCAACC AGCCTTTTCCACAAGC-3' and reverse, 5'-GACTATATG GATGCTTCCCAGTA-3'; RAR related orphan receptor $\gamma 2$ $(\mathrm{ROR} \gamma \mathrm{t})$, forward, 5'-ACCTCCACTGCCAGCTGTGTGCTG TC-3' and reverse, 5'-TCATTTCTGCACTTCTGCATGTAG ACTGTCCC-3'; ADAMTS-4, forward, 5'-TGCCGCTAAAGC CTTTAAACACAGCCA-3' and reverse, 5'-AGAAGCTGC GTAGGGTCTGG-3'; ADAMTS-5, forward, 5'-CAAGCG TTTAATGTCTTCAATCCTTA-3' and reverse, 5'-ACTGCT GGGTGGCATCGT-3'; $\beta$-actin, forward 5'-CTCCATCCT GGCCTCGCTGT-3' and reverse, 5'-GCTGTCACCTTCACC GTTCC-3'. The primers were purchased from Sangon Biotech 
Co., Ltd. (Shanghai, China). The PCR reaction conditions were: $95^{\circ} \mathrm{C}$ for $5 \mathrm{~min}$, followed by 35 cycles of denaturation at $95^{\circ} \mathrm{C}$ for $15 \mathrm{sec}$ and annealing/elongation at $55^{\circ} \mathrm{C}$ for $30 \mathrm{sec}$. Results were computed using the $2^{-\Delta \Delta \mathrm{Cq}}$ method with normalization to $\beta$-actin (18).

Western blotting. Following the aforementioned treatments, $1 \times 10^{6}$ cells were seeded in 6 -well plates and washed with ice-cold phosphate-buffered saline, and then suspended in $150 \mu \mathrm{l}$ radioimmunoprecipitation assay lysis buffer (Beyotime Institute of Biotechnology). The protein concentration was determined using a bicinchoninic acid assay kit (Beyotime Institute of Biotechnology). An equal quantity of protein $(40 \mu \mathrm{g})$ was loaded per lane and separated using $10 \%$ SDS-PAGE, followed by transfer to nitrocellulose membranes. Subsequently, the membranes were blocked with $5 \%$ skim milk for $2 \mathrm{~h}$ at room temperature, then incubated with rabbit anti-Akt (phospho S473; cat. no. ab81283), rabbit anti-p38MAPK (phospho T180 + Y182; cat. no. ab4822) or rabbit anti-Akt (cat. no. ab8805; Abcam) and rabbit anti-p38MAPK (cat. no. ab170099; all 1:1,000; Abcam) primary antibodies overnight at $4^{\circ} \mathrm{C}$. Following three washes with tris-buffered saline-Tween solution, the membranes were incubated with horseradish peroxidase-labeled goat anti-rabbit immunoglobulin G (1:5,000; cat. no. A0208; Beyotime Institute of Biotechnology) for $1 \mathrm{~h}$ at room temperature. Finally, bands were detected using enhanced chemiluminescence reagents (Wuhan Boster Biological Technology, Ltd., Wuhan, China) on Amersham Imager 600 System (GE Healthcare Life Sciences). Protein expression levels were analyzed with Image-Pro Plus software 6.0 (Media Cybernetics, Inc., Rockville, MD, USA).

Statistical analysis. All data in the present study are expressed as mean \pm standard error of the mean. Statistical analysis was conducted using SPSS 19.0 (IBM Corp., Armonk, NY, USA). Statistical significance was determined by one-way analysis of variance with Bonferroni post-hoc tests. P-values are presented for individual experiments and four repetitions of each experiment were conducted. $\mathrm{P}<0.05$ was considered to indicate a statistically significant difference.

\section{Results}

DCs generated from OA samples. Expression of co-stimulatory molecules and maturation markers (CD86, CD83, CD40 and HLA-DR) in Dex/vitD3-DCs obtained from patients with OA was determined by flow cytometry. In the presence of Dex/vitD3, the upregulation of co-stimulatory molecules induced by LPS stimulation was abrogated (Fig. 1A). Dex/vitD3-DCs expressed markedly lower CD83 than mature DCs. These findings indicated that the Dex/vitD3-DCs had a semi-mature phenotype. Similar results were observed in Dex/vitD3-DCs from normal subjects (data not shown). In order to characterize the effect of Dex/vitD3 on DC function, the generation of cytokines by DCs was analyzed. DCs induced by LPS, when compared with untreated immature DCs (the DC group), the LPS + DC group exhibited increased secretion of TNF- $\alpha(389 \pm 34$ vs. $27 \pm 9 \mathrm{pg} / \mathrm{ml}$, respectively; $\mathrm{n}=16$; $\mathrm{P}<0.01), \mathrm{IL}-1 \beta(486 \pm 35$ vs. $31 \pm 9 \mathrm{pg} / \mathrm{ml}$, respectively; $\mathrm{n}=16$;
$\mathrm{P}<0.01)$ and IL-6 (585 \pm 66 vs. $27 \pm 11 \mathrm{pg} / \mathrm{ml}$, respectively; $\mathrm{n}=16$; $\mathrm{P}<0.01$; Fig. 1B-D). A low amount of IL-10 was detected in the DC group and the LPS + DC group $(25 \pm 5$ and $34 \pm 5 \mathrm{pg} / \mathrm{ml}$, respectively; $\mathrm{n}=16)$. The $\mathrm{DC}+\mathrm{Dex} / \mathrm{vitD} 3+\mathrm{LPS}$ group significantly inhibited the production of TNF- $\alpha(107 \pm 14 \mathrm{pg} / \mathrm{ml})$, IL-1 $\beta(102 \pm 20 \mathrm{pg} / \mathrm{ml})$ and IL-6 $(135 \pm 32 \mathrm{pg} / \mathrm{ml} ; \mathrm{n}=16 ; \mathrm{P}<0.01$; Fig. 1B-D). However, IL-10 (202 \pm 17 vs. $26 \pm 5$ pg/ml, respectively; $\mathrm{n}=16$; $\mathrm{P}<0.01$; Fig. $1 \mathrm{E}$ ) significantly increased secretion in the Dex/vitD3 + DC group compared with the DC group. Additionally, IL-10 production in the DC + Dex/vitD3 + LPS group was significantly inhibited compared with the $\mathrm{DC}+$ Dex/vitD3 group $(58 \pm 11 \mathrm{pg} / \mathrm{ml} ; \mathrm{n}=16 ; \mathrm{P}<0.01)$. Notably, compared with the DC group, the LPS + DC group slightly and significantly increased TGF- $\beta(62 \pm 14$ vs. $26 \pm 8 \mathrm{pg} / \mathrm{ml}$, respectively; $\mathrm{n}=16 ; \mathrm{P}<0.05)$ secretion, and Dex/vitD3 potentiated this production to $190 \pm 31 \mathrm{pg} / \mathrm{ml}$ compared with the LPS + DC group (n=16; $\mathrm{P}<0.01$; Fig. $1 \mathrm{~F})$.

Dex/vitD3-DCs attenuate autologous $T$ cell proliferation. To assess the proliferation of autologous $\mathrm{T}$ cells when cultured with Dex/vitD3-DCs, the thymidine incorporation assay were performed. Compared with DC-T cell co-cultures, LPS + DC increased autologous $\mathrm{T}$ cell proliferation $(\mathrm{n}=6 ; \mathrm{P}<0.01$; Fig. 1G). When compared with the LPS + DC group, the proliferation of autologous $\mathrm{T}$ cell in the DC+Dex/vitD3+LPS group was signifcantly reduced $(n=6 ; P<0.01$; Fig. $1 \mathrm{G})$.

Dex/vitD3-DCs reduce the differentiation of naive $T$ cells to Th1 and Th17 cells, and induce differentiation to IL-10 producing cells. The mRNA level of transcription factors that serve as markers of Th1 (T-bet), Th2 (Gata-3), Th17 (ROR $\gamma \mathrm{t})$ and Treg (Foxp3) cell differentiation were determined by RT-qPCR in DC-primed naive T cells. Compared with $\mathrm{T}$ cells stimulated by mature DCs (DC + LPS), T cells primed by $\mathrm{DC}+$ Dex/vitD3 + LPS exhibited significantly lower T-bet ( $n=6 ; \mathrm{P}<0.01$; Fig. 2A). No significance was identified in Gata3 mRNA expression between the DC, DC + LPS and $\mathrm{DC}+$ Dex/vitD3 + LPS groups (n=6; Fig. 2B). Compared with the DC + LPS group, the DC + Dex/vitD3 + LPS group exhibited significantly lower ROR $\gamma t$ mRNA expression and higher Foxp3 levels $(n=6 ; \mathrm{P}<0.01$; Fig. $2 \mathrm{C}$ and D). Compared with the DC group, intracellular staining of T cells following stimulation with DC + LPS revealed a considerable proportion of the T-cells were IFN- $\gamma$ - and IL-17-producing cells $(n=6 ; P<0.01$; Fig. $2 \mathrm{E}$ and F). When Dex/vitD3 were added in the DC + LPS group, the percentage of IL-10- and FoxP3-producing cells decreased significantly compare with the $\mathrm{DC}+\mathrm{Dex} /$ vitD3 group $(\mathrm{n}=6 ; \mathrm{P}<0.01)$. Compared with $\mathrm{T}$ cells in the DC group, T cells stimulated by DC + Dex/vitD3, termed $\mathrm{T}[\mathrm{DC}+\mathrm{Dex} / \mathrm{vitD} 3]$, contained a significantly greater proportion of IL-10-producing cells $(\mathrm{n}=6 ; \mathrm{P}<0.01)$ and had slightly and significantly increased expression of Foxp3 ( $n=6 ; \mathrm{P}<0.01)$. However, the DC + Dex/vitD3 + LPS group also exhibited a decreased proportion of IL-10- and Foxp3-producing T cells compared with the DC + Dex/vitD3 group ( $\mathrm{n}=6$; $\mathrm{P}<0.01$; Fig. $2 \mathrm{E}$ and $\mathrm{F}$ ). These results demonstrated that OA Dex/vitD3-DCs inhibited the activation and proliferation of naive $\mathrm{T}$ cells. Naive $\mathrm{T}$ cells differentiated into IL-10-producing effector cells and the pro-inflammatory phenotype was reduced. 
A

DC - DC +Dex/vitD3 - DC +LPS - DC +Dex/vitD3 +LPS
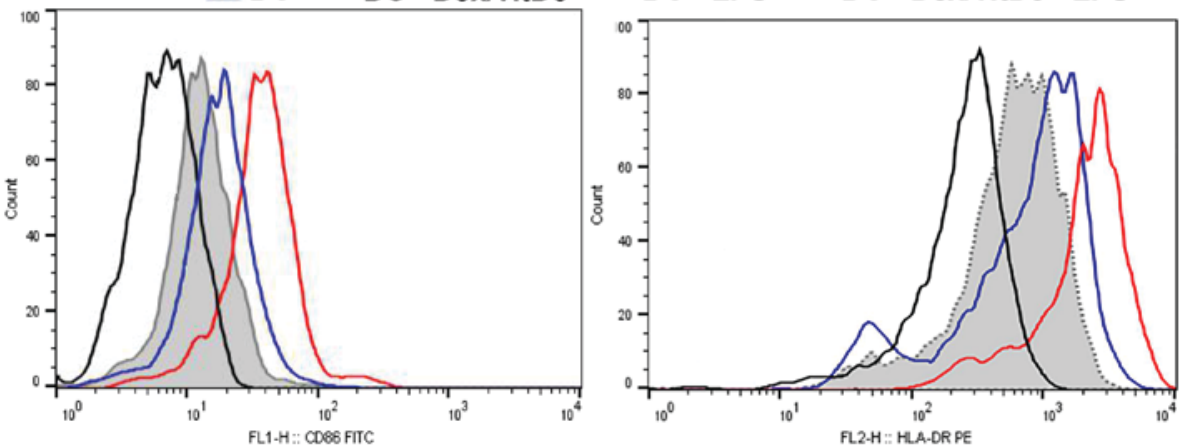

CD86

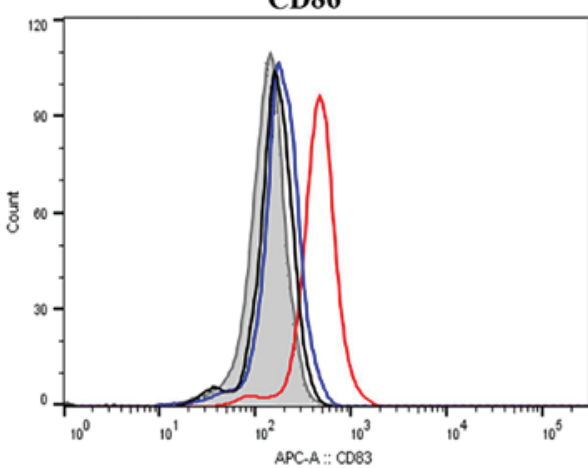

CD83

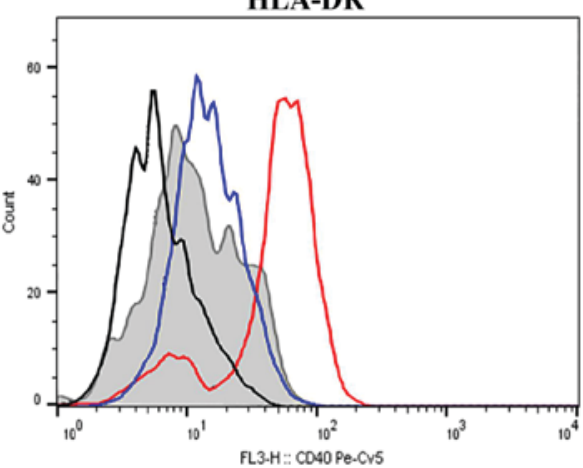

CD40
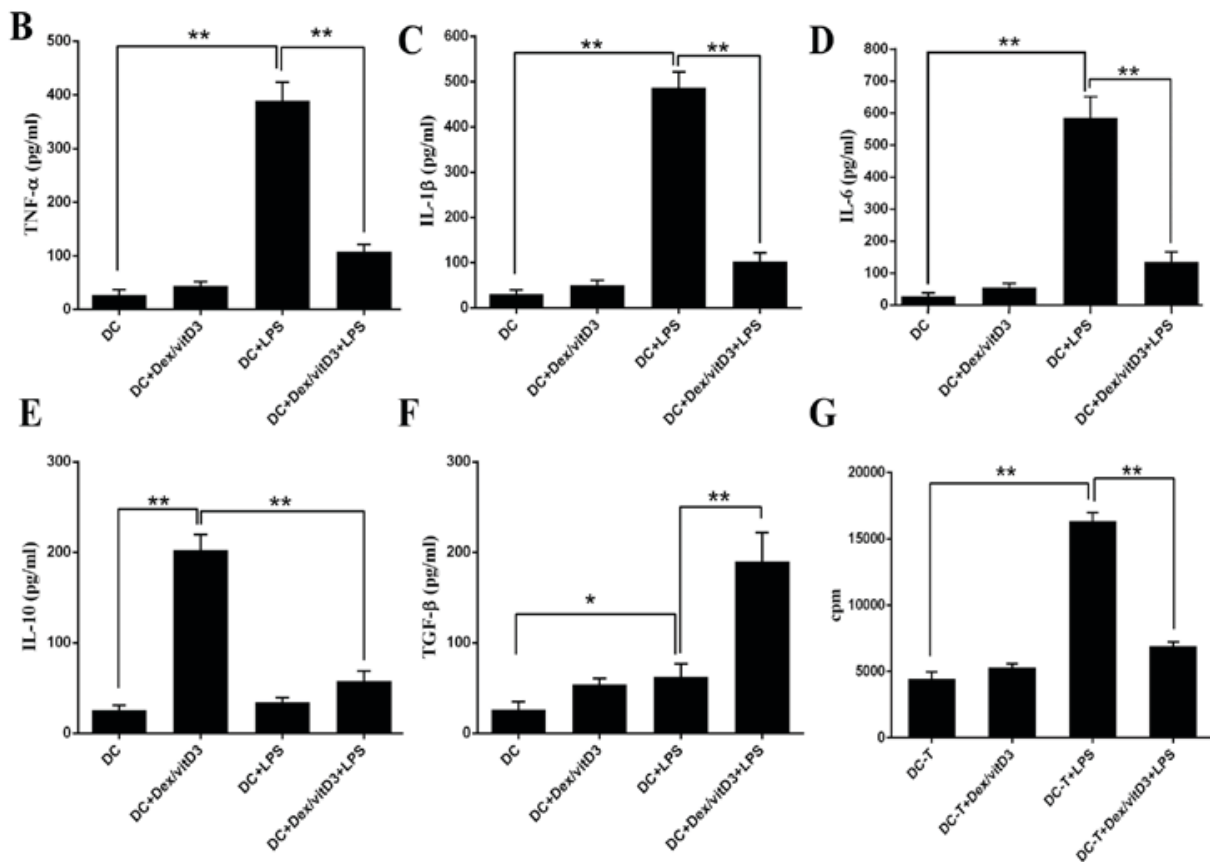

Figure 1. DCs increase cytokine production and attenuate T cell proliferation. (A) Expression of activation and maturation markers: CD86, HLA-DR, CD83 and CD40. The flow diagrams show data from one representative of 16 independent experiments. (B-F) Cytokine production was determined using ELISA. Results are presented as the mean concentration \pm standard error of the mean of 16 independent experiments. (G) Autologous T cells proliferative response was assessed by cpm of $\left[{ }^{3} \mathrm{H}\right]$-thymidine incorporation. A representative experiment out of six is presented. ${ }^{*} \mathrm{P}<0.05$ and ${ }^{* * *} \mathrm{P}<0.01$. DC, dendritic cell; $\mathrm{HLA}-\mathrm{DR}$, human leukocyte antigen-antigen D related; CD, cluster of differentiation; cpm, counts per minute; Dex, dexamethasone; vitD3, vitamin D3; LPS, lipopolysaccharide.

Dex/vitD3-DCs induce IL-10-producing Tcells withregulatory functions. Following the addition of T [DC + Dex/vitD3 + LPS] cells in a 10:1 ratio, the proliferation of naive $\mathrm{CD} 4^{+} \mathrm{T}$ cells stimulated with mature DCs was markedly decreased compared with the T-DC group $(n=6 ; P<0.01 ;$ Fig. 3A). Therefore, T cells stimulated with LPS + DC inhibited the responses of naive T cells. The addition of neutralizing anti-IL-10R or anti-IL-10 monoclonal antibodies diminished the suppression on $\mathrm{T}$ cell proliferation caused by T-DC [Dex/vitD3 + LPS] cells compared with the T-DC (Dex/vitD3 + LPS)+IL-10R group or the T-DC (Dex/vitD3 + LPS) + IL-10 group (P<0.01; Fig. 3B). In addition, T-DC [Dex/vitD3 + LPS] cells required cell-cell contact for their suppressive activity, which was confirmed in the thymidine incorporation assays $(n=6 ; P<0.01$; Fig. $3 C)$. 

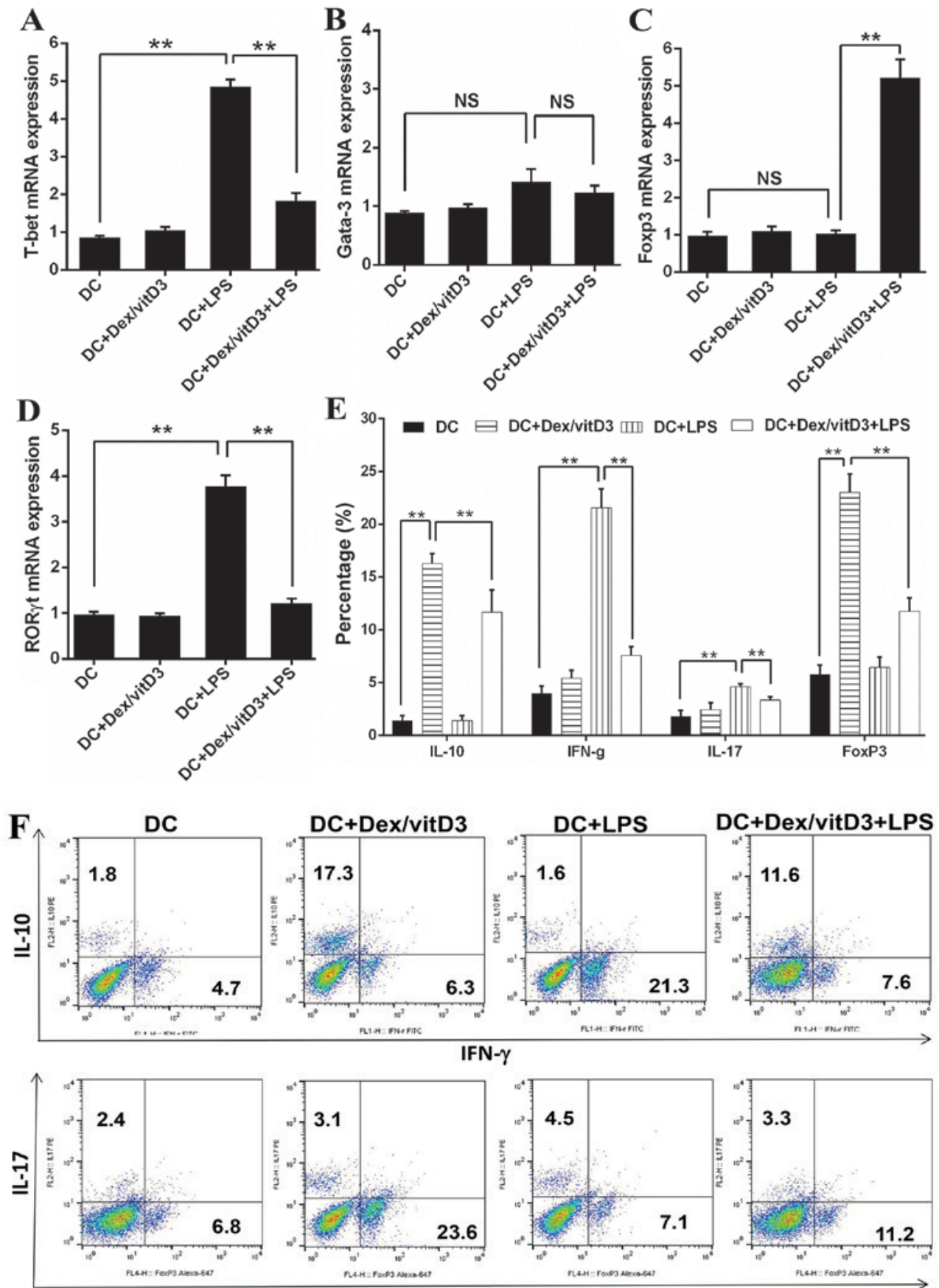

FoxP3

Figure 2. Dex/vitD3-treated DCs induce the generation of IL-10-producing T cells. (A-D) The mRNA levels of T cell polarized transcription factors (including T-bet, Gata-3, ROR $\gamma$ t and Foxp3) were detected. Results are presented as the mean concentration \pm standard error of the mean of six independent experiments. (E) Summary of six independent experiments. (F) Intracellular staining of cytokine production assessed by flow cytometry. These data provided the percentage of IFN- $\gamma$-, IL-17- and IL-10-producing cells in T [DC], T [DC + LPS] or T [DC + Dex/vitD3 + LPS] cells. A representative experiment out of six is presented. ${ }^{* *} \mathrm{P}<0.01$. Dex, dexamethasone; vitD3, vitamin D3; DC, dendritic cell; IL, interleukin; T-bet, T-box 21; Gata-3, GATA binding protein 3; ROR $\gamma \mathrm{t}$, RAR related orphan receptor $\gamma$ 2; Foxp3, forkhead box P3; LPS, lipopolysaccharide; IFN- $\gamma$, interferon- $\gamma$; T, T cells.

Together, these results indicated that $\mathrm{T}$ cells stimulated by Dex/vitD3-DCs exhibited function equivalent to that of Tregs.

Dex/vitD3-DCs (tolDCs) and IL-10-producing Tregs repress the production of MMPs by chondrocytes. Toluidine blue and II-collagen antibody staining of cartilage cells was performed, resulting in light blue and brown chondrocyte staining (Fig. 4A). To characterize the effects of Dex/vitD3-DCs and IL-10-producing Tregs on chondrocytes,
TNF- $\alpha$-pretreated chondrocytes were co-cultured with the autologous Dex/vitD3-DCs (considered tolDCs) or T $[\mathrm{DC}+$ Dex/vitD3 + LPS) cells (considered Tregs). TNF- $\alpha$ induction significantly increased secretion of MMP-1 and MMP-13 ( $n=8 ; \mathrm{P}<0.01)$ compared with untreated chondrocytes (Fig. 4B). TolDCs and Tregs inhibited the production of MMP-1 and MMP-13 $(n=8 ; \mathrm{P}<0.01)$ in chondrocyte cultures compared with the TNF- $\alpha$-induced levels (Fig. 4B). Furthermore, mRNA levels of ADAMTS-4 and ADAMTS-5 were determined by 

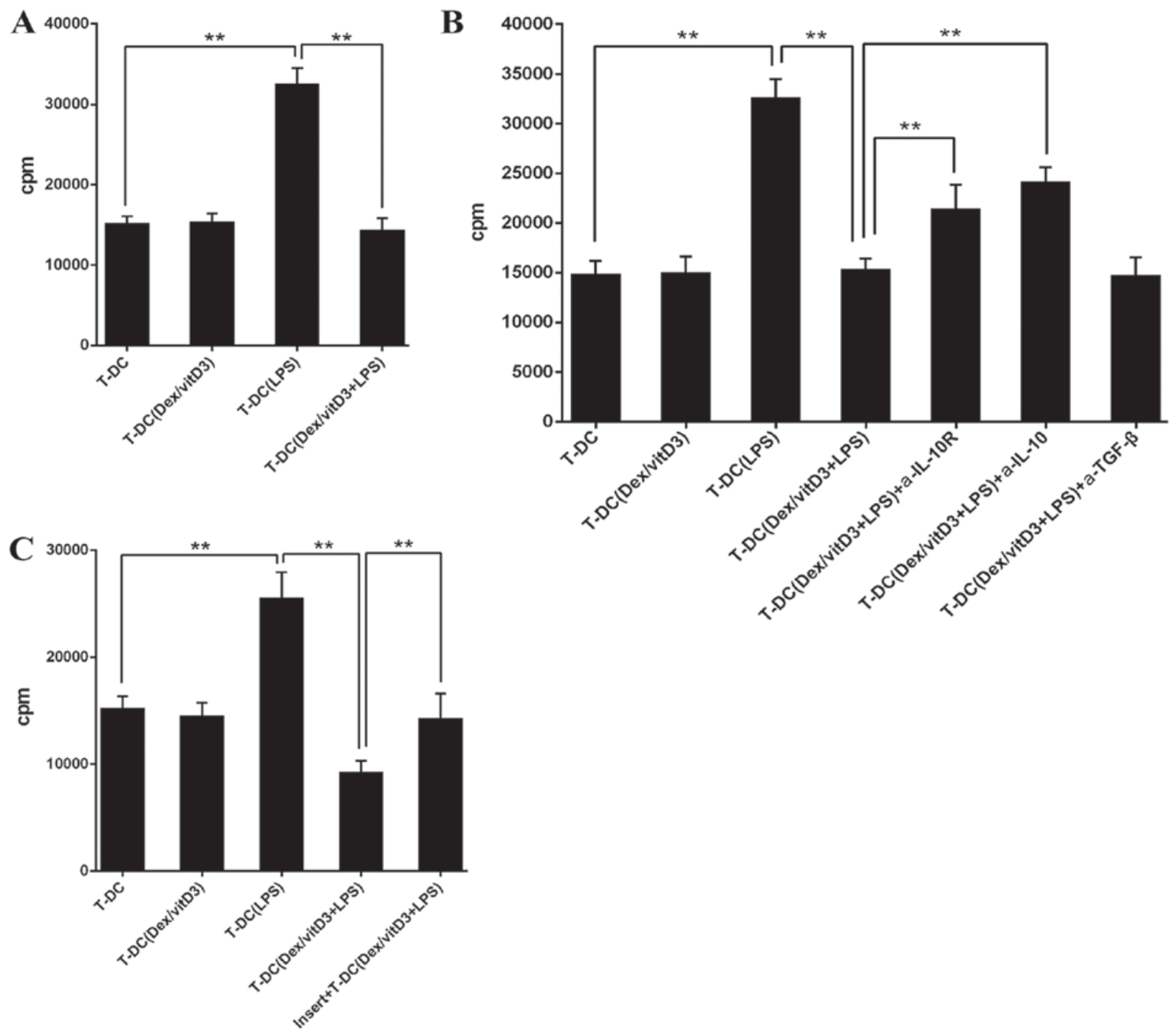

Figure 3. T [DC + Dex/vitD3 + LPS] cells inhibit primary T cell proliferation. (A) Proliferation of naive CD4+ T cells was markedly decreased. Results show a representative experiment out of six. (B) Anti-IL-10R or anti-IL-10 diminished the suppression on T cell proliferation. Results show a representative experiment of six. (C) T [DC + Dex/vitD3 + LPS] cell-mediated inhibition required cell-cell contact. Results show a representative experiment of six. ${ }^{* *} \mathrm{P}<0.01$. $\mathrm{T}$, T cells; DC, dendritic cell; Dex, dexamethasone; vitD3, vitamin D3; LPS, lipopolysaccharide; IL-10, interleukin 10; IL-10R, interleukin 10 receptor; TGF- $\beta$; transforming growth factor- $\beta$; a-, anti-.

RT-qPCR. TNF- $\alpha$ significantly increased the expression of ADAMTS-4 and ADAMTS-5 compared with those in the untreated chondrocytes group. Chondrocytes co-cultured with tolDCs and Tregs exhibited marked reduction in ADAMTS-4 and ADAMTS-5 $(\mathrm{n}=8 ; \mathrm{P}<0.01)$ compared with those treated with TNF- $\alpha$ (Fig. 4C).

Akt and 38 MAPK phosphorylation are involved in the effect of Dex/vitD3. The underlying mechanisms of tolerogenicity in Dex/vitD3-DCs were investigated. The phosphorylated forms of Akt and p38 MAPK were detected by phospho flow. LPS-induced phosphorylation of Akt and p38 MAPK was reduced by Dex and vitD3 (Fig. 5A). Additionally, compared with the DC group, the DC + LPS group exhibited significantly increased phosphorylation of Akt and p38MAPK protein, $(\mathrm{n}=6 ; \mathrm{P}<0.01$; Fig. 5B and C). However, compared with the DC + LPS group, the DC + Dex/vitD3 + LPS exhibited significantly reduced Akt and p38MAPK protein phosphorylation levels $(n=6 ; P<0.01$; Fig. $5 B$ and $C)$.

\section{Discussion}

Dex/vitD3-DCs were generated using PBMCs from patients with OA. The PBMCs were treated with Dex and vitD3, which was followed by LPS-induced differentiation in vitro. Dex increases the expression of the vitamin $\mathrm{D}$ receptor, which makes $\mathrm{T}$ cells more susceptible to vitD3 stimulation $(15,16)$. In the current study, the expression of the vitamin $\mathrm{D}$ receptor on DCs was increased following the treatment with Dex (data not shown). Additionally, as reportedly previously, Dex/vitD3-DCs produced from patients with OA exhibited low expression of co-stimulatory molecules, MHC class II and maturation markers, compared with tolDCs derived from healthy volunteers (19), and OA Dex/vitD3-DCs attenuated autologous $\mathrm{T}$ cell activation and proliferation.

Naive $T$ cells that were exposed to OA Dex/vitD3-DCs exhibited a less pro-inflammatory phenotype and reduced production of IFN- $\gamma$ and IL-17. The low level of IL- 6 produced by Dex/vitD3-DCs likely facilitated the differentiation of Tregs 
$\mathbf{A}$
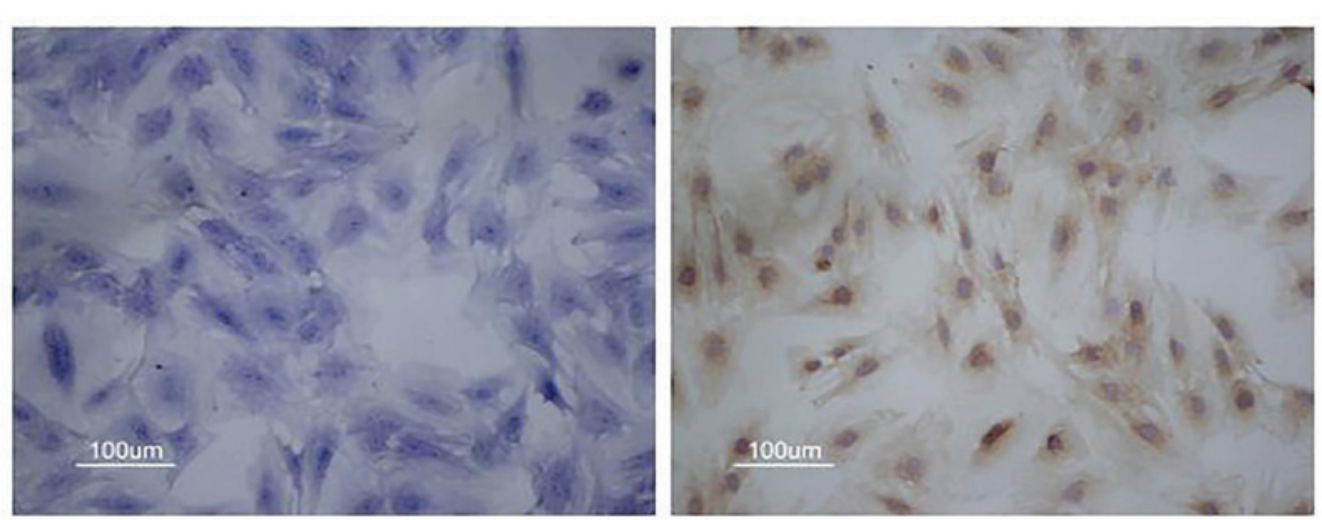

B

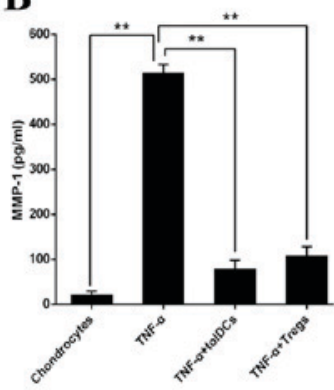

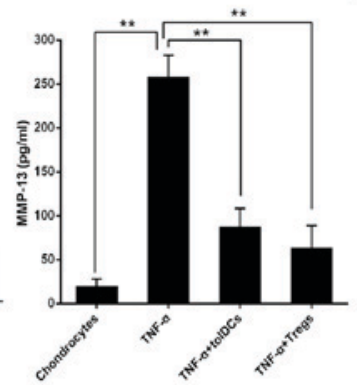

C

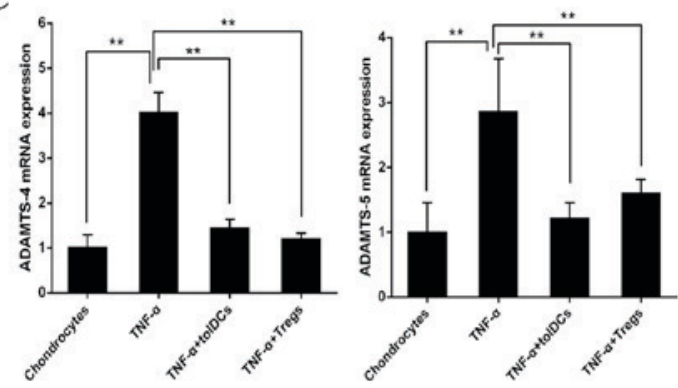

Figure 4. Dex/vitD3-treated DCs and IL-10-producing T regulatory cells inhibit the degradation of extracellular cartilage matrix of chondrocytes. (A) Chondrocytes were identified by toluidine blue and II-collagen antibody staining (magnification, x200). (B) MMP-1 and MMP-13 production determined by ELISA. Results are presented as the mean \pm standard error of the mean of eight independent experiments. (C) mRNA levels of ADAMTS-4 and ADAMTS-5 determined by reverse transcription-quantitative polymerase chain reaction. Results are presented as the mean \pm standard error of the mean of eight independent experiments. ${ }^{* *} \mathrm{P}<0.01$. Dex, dexamethasone; vitD3, vitamin D3; DC, dendritic cell; IL-10, interleukin 10; MMP, matrix metalloproteinase; ADAMTS, A disintegrin and metalloproteinase with thrombospondin motifs.

A -.. DC DC +Dex/vitD3

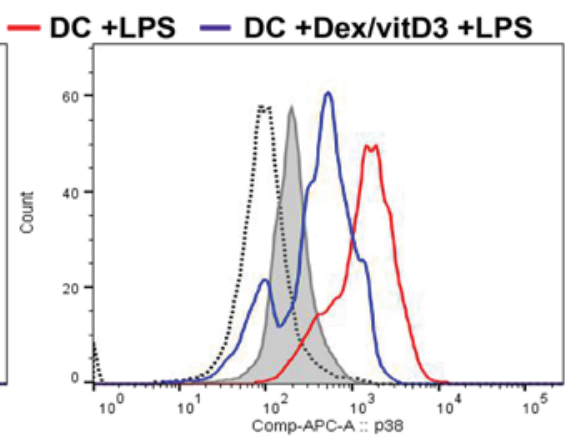

p-AKT
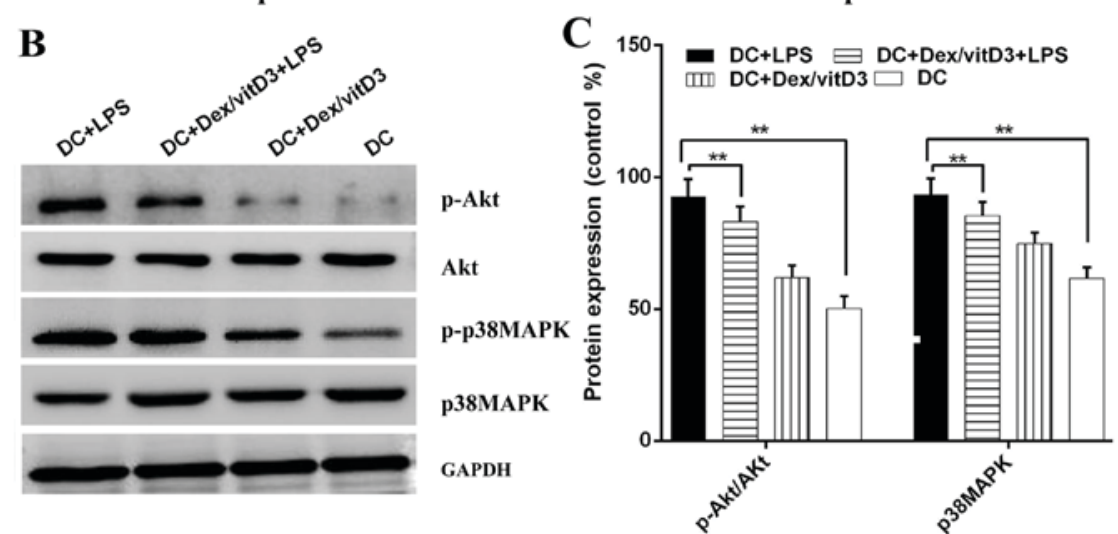

Figure 5. Role of the Akt/p38 pathway in the effect of Dex/vitD3-treated DCs. (A) The phospho flow diagrams present data from one representative of six independent experiments. (B) Changes in the levels of p-Akt and p-p38 MAPK expression following the different treatments. (C) Densitometric analysis of p-Akt and p-p38 MAPK. ${ }^{*} \mathrm{P}<0.01$. Akt, protein kinase B; Dex, dexamethasone; vitD3, vitamin D3; DC, dendritic cell; MAPK, mitogen-activated protein kinase; LPS, lipopolysaccharide; p-, phosphorylated. 
into Th17 cells (20). Additionally, differentiation of T cells to IL-10-generating Tregs appeared to be induced by the vitD3/Dex, and these cells suppressed autologous $\mathrm{T}$ cell activation and proliferation. Notably, the IL-10-producing Tregs, which had increased expression of Foxp3, were different from type 1 Tregs described previously $(19,21,22)$. IL-10 production and cell-cell contact were required to mediate the regulatory functions of $\mathrm{T}$ cells in the present study. To investigate the molecular mechanisms involved in the effect of Dex/vitD3, several signaling pathways, including the phosphoinositide 3-kinase (PI3K), p38 MAPK and extracellular signal-regulated kinase $1 / 2$ pathways, have been investigated (11). In line with recent findings (23), the results of the current study demonstrated that the p38 MAPK was involved in the effects of Dex/vitD3. PI3K signaling may also serve a function in Dex/vitD3-induced effects (24).

OA, the most common joint disease worldwide, is a progressive inflammatory disease characterized by cartilage destruction, synovitis and subchondral bone reconstruction $(3,11)$. Changes in innate and adaptive immunity also appear to be involved in OA, as activated T cells are present in the synovial tissue of articular specimens from patients with OA (25). In fact, study has indicated that immune-mediated inflammation is common in OA synovial tissue, where immune cell infiltration and cytokine secretion are prominent manifestations (26). Pro-inflammatory cytokines, including IL-1 $\beta$, TNF- $\alpha$ and IL-6, are involved in the pathophysiology of OA. IL-1 $\beta$ levels are generally elevated in OA synovial fluid (17). The levels of serum IL- 6 and C-reactive protein are increased, and consistently higher in individuals diagnosed with radiographic knee OA than in healthy controls (27). Furthermore, the levels of IL- 6 and TNF- $\alpha$ in serum have been previously reported to be associated with loss of cartilage in elderly patients with OA (28). Barker et al (29) reported that early $\mathrm{OA}$ of the knee was associated with increased serum levels of TNF- $\alpha$, IL-5, IL-6 and IL-12. Additionally, the level of IL-17 has been demonstrated to be higher in serum and synovial fluid of patients with OA compared with controls (30).

Pro-inflammatory cytokines that stimulate the expression of MMPs and ADAMTS proteins in human chondrocytes cultures are also significantly elevated in the synovial fluid of patients with OA (31). Osteoarthritic cartilage degradation initiates structural changes within the joints, which can considerably impact joint function (3). The loss of chondrocyte extracellular matrix proteins, collagen II and aggrecan, is a hallmark of OA (32). ADAMTS-4 and ADAMTS-5 have an important role in the degradation of aggrecan in cartilage, which occurs early in OA pathogenesis (33). MMPs are enzymes that can degrade collagen II, and are secreted in response to various stimuli, including cytokines and growth factors, in arthritic joints (34).

To the best of our knowledge, the findings of the current study are the first to demonstrate the generation of tolDCs from patients with OA by stimulation with vitD3 and dex. Each of vitD3 and Dex have been previously shown to induce tolDCs $(21,22)$ and may extend the survival time of organ allografts (35). Indeed, Dex/vitD3-stimulated DCs exhibited better tolerance than using vitD3 or Dex individually (36), or other pharmacological agents (19). It has been reported that Dex/vitD3-DCs have beneficial therapeutic effects in antigen-specific models, including collagen-induced arthritis (30). Considering the important role of $\mathrm{T}$ cells and chondrocytes in the pathogenesis of OA (37), the present study demonstrated the beneficial immunomodulatory effects of Dex/vitD3-DCs on T cells from patients with OA, with reduced production of Th1 and Th17 cytokines in the pro-inflammatory profile. Both the Dex/vitD3-treated DCs, and the subsequently generated IL-10-producing Tregs, exhibited a protective effect on chondrocytes, suppressing the expression of ADAMTS-4 and ADAMTS-5 and reducing the production of MMP-1 and MMP-13. Therefore, combination of vitD3 and Dex followed by LPS maturation may be an effective strategy for induction of tolDCs in patients with OA, and thus have therapeutic potential.

\section{Acknowledgements}

Not applicable.

\section{Funding}

The present study was supported by grants from the National Natural Science Foundation of China (grant no. 81373421) and Anhui Province Natural Science Youth Funding Projects (grant no. 1808085QH241).

\section{Availability of data and materials}

The datasets used and/or analyzed during the current study are available from the corresponding author on reasonable request.

\section{Authors' contributions}

GW performed the statistical analysis and wrote the manuscript. YF extracted peripheral blood from the patients, and separated autologous $\mathrm{T}$ cell and naive T cells. JZ performed the co-cultured cells experiments and submitted the manuscript. WC performed the experiments. BX and $\mathrm{XC}$ designed the study and revised the manuscript.

\section{Ethics approval and consent to participate}

Clinical and laboratory examinations were performed after obtaining informed written consent from all patients and approval from the Ethics Committee of Anhui Medical University, Hefei, China.

\section{Patient consent for publication}

Not applicable.

\section{Competing interests}

The authors declare that they have no competing interests.

\section{References}

1. Felson DT: Clinical practice. Osteoarthritis of the knee. N Engl J Med 354: 841-848, 2006. 
2. Hügle T, Geurts J,Nüesch C, Müller-Gerbl M and Valderrabano V: Aging and osteoarthritis: An inevitable encounter? J Aging Res 2012: 950192, 2012.

3. Pelletier JP, Martel-Pelletier J and Abramson SB: Osteoarthritis, an inflammatory disease: Potential implications for the selection of new therapeutic targets. Arthritis Rheum 44: 1237-1247, 2001.

4. Goode AP, Nelson AE, Kraus VB, Renner JB and Jordan JM Biomarkers reflect differences in osteoarthritis phenotypes of the lumbar spine: The Johnston County Osteoarthritis Project. Osteoarthritis Cartilage 25: 1672-1679, 2017.

5. Delco ML, Bonnevie ED, Szeto HS, Bonassar LJ and Fortier LA Mitoprotective therapy preserves chondrocyte viability and prevents cartilage degeneration in an ex vivo model of posttraumatic osteoarthritis. J Orthop Res, 2018.

6. Roos EM and Arden NK: Strategies for the prevention of knee osteoarthritis. Nat Rev Rheumatol 12: 92-101, 2016.

7. Hulet C, Menetrey J, Beaufils P, Chambat P, Djian P, Hardy P, Potel JF, Servien E and Seil R; French Arthroscopic Society (SFA): Clinical and radiographic results of arthroscopic partial lateral meniscectomies in stable knees with a minimum follow up of 20 years. Knee Surg Sports Traumatol Arthrosc 23: 225-231, 2015.

8. Verma P and Dalal K: ADAMTS-4 and ADAMTS-5: Key enzymes in osteoarthritis. J Cell Biochem 112: 3507-3514, 2011.

9. Wang P, Ying J, Luo C, Jin X, Zhang S, Xu T, Zhang L, Mi M Chen D, Tong $\mathrm{P}$ and Jin $\mathrm{H}$ : Osthole promotes bone fracture healing through activation of BMP signaling in chondrocytes. Int J Biol Sci 13: 996-1007, 2017.

10. Wang $\mathrm{T}$ and He C: Pro-inflammatorycytokines: The link between obesity andosteoarthritis. Cytokine Growth Factor Rev: S1359-6101(18)30119-9, 2018.

11. Kapoor M, Martel-Pelletier J, Lajeunesse D, Pelletier JP and Fahmi H: Role of proinflammatory cytokines in the pathophysiology of osteoarthritis. Nat Rev Rheumatol 7: 33-42,2011.

12. Chen YH, Yu Z, Fu L, Wang H, Chen X, Zhang C, Lv ZM and Xu DX: Vitamin D3 inhibits lipopolysaccharide-induced placental inflammation through reinforcing interaction between vitamin D receptor and nuclear factor kappa B p65 subunit. Sci Rep 5: 10871, 2015.

13. Anderson AE, Swan DJ, Sayers BL, Harry RA, Patterson AM, von Delwig A, JH Robinson, Isaacs JD and Hilkens CM: LPS activation is required for migratory activity and antigen presentation by tolerogenic dendritic cells. J Leukoc Biol 85: 243-250, 2009.

14. Feili-Hariri M, Dong X, Alber SM, Watkins SC, Salter RD and Morel PA: Immunotherapy of NOD mice with bone marrow-derived dendritic cells. Diabetes 48: 2300-2308, 1999.

15. Charbonnier LM, Van Duivenvoorde LM, Apparailly F, Cantos C, Han WG, Noël D, Duperray C, Huizinga TW, Toes RE, Jorgensen C and Louis-Plence P: Immature dendritic cells suppress collagen-induced arthritis by in vivo expansion of CD49b+ regulatory T cells. J Immunol 177: 3806-3813, 2006.

16. Lutz MB and Schuler G: Immature, semi-mature and fully mature dendritic cells: Which signals induce tolerance or immunity? Trends Immunol 23: 445-449, 2002.

17. Malemud CJ: Anticytokine therapy for osteoarthritis: Evidence to date. Drugs Aging 27: 95-115, 2010.

18. Livak KJ and Schmittgen TD: Analysis of relative gene expression data using real-time quantitative PCR and the 2(-Delta Delta C(T)) method. Methods 25: 402-408, 2001.

19. Volchenkov R, Karlsen M, Jonsson R and Appel S: Type 1 regulatory $\mathrm{T}$ cells and regulatory $\mathrm{B}$ cells induced by tolerogenic dendritic cells. Scand J Immunol 77: 246-254, 2013.

20. Kimura A and Kishimoto T: IL-6: Regulator of Treg/Th17 balance. Eur J Immunol 40: 1830-1835, 2010.

21. Unger WW, Laban S, Kleijwegt FS, Van Der Slik AR and Roep BO: Induction of Treg by monocyte-derived DC modulated by vitamin D3 or dexamethasone: Differential role for PD-L1. Eur J Immunol 39: 3147-3159, 2009.
22. Xia CQ, Peng R, Beato F and Clare-Salzler MJ: Dexamethasone induces IL-10-producing monocyte-derived dendritic cells with durable immaturity. Scand J Immunol 62: 45-54, 2005.

23. Dáňová K, Klapetková A, Kayserová J, Šedivá A, Špíšek R and Jelínková LP: NF-kB, p38 MAPK, ERK1/2, mTOR, STAT3 and increased glycolysis regulate stability of paricalcitol/dexamethasone-generated tolerogenic dendritic cells in the inflammatory environment. Oncotarget 6: 14123-14138, 2015.

24. Yang J, Chen Q, Tian S, Song S, Liu F, Wang Q and Fu Z: The role of 1,25-dyhydroxyvitaminD3in mouse liver ischemia reperfusion injury: Regulation of autophagy through activation of MEK/ERK signaling and PTEN/PI3K/Akt/mTORC1 signaling. Am J Transl Res 7: 2630-2645, 2015.

25. Anderson AE, Sayers BL, Haniffa MA, Swan DJ, Diboll J, Wang XN, Isaacs JD and Hilkens CM: Differential regulation of naive and memory CD4+ T cells by alternatively activated dendritic cells. J Leukoc Biol 84: 124-133, 2008.

26. Banchereau J, Pascual V and Palucka AK: Autoimmunity through cytokine-induced dendritic cell activation. Immunity 20 : 539-550, 2004

27. Livshits G, Zhai G, Hart DJ, Kato BS, Wang H, Williams FM and Spector TD: Interleukin-6 is a significant predictor of radiographic knee osteoarthritis: The Chingford Study. Arthritis Rheum 60: 2037-2045, 2009.

28. Stannus O, Jones G, Cicuttini F, Parameswaran V, Quinn S, Burgess J and Ding C: Circulating levels of IL-6 and TNF- $\alpha$ are associated with knee radiographic osteoarthritis and knee cartilage loss in older adults. Osteoarthritis Cartilage 18: 1441-1447, 2010.

29. Barker T, Rogers VE, Henriksen VT, Aguirre D, Trawick RH, Rasmussen GL and Momberger NG: Serum cytokines are increased and circulating micronutrients are not altered in subjects with early compared to advanced knee osteoarthritis. Cytokine 68: 133-136, 2014.

30. Chen B, Deng Y, Tan Y, Qin J and Chen LB: Association between severity of knee osteoarthritis and serum and synovial fluid interleukin 17 concentrations. J Int Med Res 42: 138-144, 2014.

31. Su YP, Chen CN, Huang KC, Chang HI, Lee KC, Lo CM and Chang SF: Leptin induces MMP1/13 andADAMTS4 expressions through bone morphogenetic protein-2 autocrine effect in human chondrocytes. J Cell Biochem 119: 3716-3724, 2018.

32. Wieland HA, Michaelis M, Kirschbaum BJ and Rudolphi KA: Osteoarthritis-an untreatable disease? Nat Rev Drug Discov 4: 331-344, 2005.

33. Lee AS, Ellman MB, Yan D, Kroin JS, Cole BJ, van Wijnen AJ and Im HJ: A current review of molecular mechanisms regarding osteoarthritis and pain. Gene 527: 440-447, 2013.

34. Vincenti MP and Brinckerhoff CE: Transcriptional regulation of collagenase (MMP-1, MMP-13) genes in arthritis: Integration of complex signaling pathways for the recruitment of gene-specific transcription factors. Arthritis Res 4: 157-164, 2002.

35. Emmer PM, van der Vlag J, Adema GJ and Hilbrands LB: Dendritic cells activated by lipopolysaccharide after dexamethasone treatment induce donor-specific allograft hyporesponsiveness. Transplantation 81: 1451-1459, 2006.

36. Ferreira GB, Kleijwegt FS, Waelkens E, Lage K, Nikolic T, Hansen DA, Workman CT, Roep BO, Overbergh L and Mathieu C: Differential protein pathways in 1,25-dihydroxyvitamin $\mathrm{d}(3)$ and dexamethasone modulated tolerogenic human dendritic cells. J Proteome Res 11: 941-971, 2012.

37. Bian Q, Wang YJ, Liu SF and Li YP: Osteoarthritis: Genetic factors, animal models, mechanisms, and therapies. Front Biosci 4: 74-100, 2012.

This work is licensed under a Creative Commons Attribution-NonCommercial-NoDerivatives 4.0 International (CC BY-NC-ND 4.0) License. 